\title{
TOWARDS A DISTRIBUTED PROCESS EXECUTION PLATFORM FOR COLLABORATIVE NETWORKS
}

\author{
A. Luis Osório ', Luis M. Camarinha-Matos ${ }^{2}$ \\ 'Instituto Superior de Engenharia de Lisboa, PORTUGAL, aosorio@deetc.isel.ipl.pt \\ ${ }^{2}$ New University of Lisbon, PORTUGAL, cam@uninova.pt
}

\begin{abstract}
Enterprise collaboration typically occurs in the context of a process. Therefore, in order to support collaboration, there is a need to establish a framework able to handle a diversity of collaborative processes with different computational and communication requirements. This paper discusses the need for such framework, points out key requirements and proposes a strategy for a supporting technological infrastructure. Processes are seen as the central modeling entity in this framework. Portability of process definitions among different technological bindings is also a considered issue.
\end{abstract}

\section{INTRODUCTION}

Computer supported collaboration is being pursued in a large diversity of application domains. For instance, there are research groups addressing collaboration in the education area, the computer supported collaborative learning (CSCL) (Dillenbourg et al., 1995), in the collaborative engineering (CE) area, in the virtual enterprises area, and in many other domains. The ECOLEAD project (Camarinha-Matos et al., 2005) is an important effort to understand collaborative ecosystems by defining the foundations and mechanisms for establishing an advanced network-based industry society. There is however a lack of a generic platform to support collaboration activities along their life cycle. From a company's point of view, collaboration acts occur many times in different contexts. They can occur during a phone-call between employees of two companies or they can occur through e-mail exchanges or through other communication forms. In terms of modeling, there is a need to represent different collaboration acts and events independently of them being based on computational transactions or human conversation or through any other channel.

The traditional workflow based approaches to enterprise process automation show various limitations when it comes to model collaboration acts occurring in the enterprise and involving or not business partners. For instance, the concept of work list and work item used to model user interactions requires a more flexible approach. An enterprise user (under some role) might need to contribute to some activity by accessing a dedicated application that is invoked by a process execution kernel 
(orchestration) following a rather different strategy than the one supported by the worklist/workitem concepts defined by WfMC (Hollingsworth, 1995).

There is a growing awareness among researchers and information and technology practitioners on the need to evolve to scenarios where existing technologies, strategies and tools cope with new challenges for enterprise collaboration. The outlining of key research challenges and reference projects (Camarinha-Matos and Afsarmanesh, 2004) is an important step to the consolidation of the area of collaborative networked organizations (CNOs). CNOs have been addressing different collaboration patterns that can emerge from different breeding environments. A breeding environment offers the necessary preparedness stage for enterprises, organizations or individuals to participate in different collaboration patterns such as virtual enterprise/virtual organization (VE/VO), professional virtual community (PVC), and even e-science and e-government forms (Camarinha-Matos and Afsarmanesh, 2004). The collaboration among members of a $\mathrm{CNO}$ needs preparation, trust, and above all, a framework making possible the management of the life cycle of the collaborative activities.

This paper discusses some of these challenges and proposes a strategy for such a collaborative framework. It is assumed that collaboration is modeled by a process that is executed on a distributed platform involving those members that were selected from a breeding environment to form some collaboration pattern. This work is based on practical experience acquired with two application cases, one in the transport systems sector and addressing a holistic approach to the collaborative electronic toll collection (ETC) management system, and another case in the public administration sector aiming to implement an integrated service to citizens in a collaboration scenario involving several public entities. In both cases a service oriented approach is adopted and a key challenge is to contribute to a technological platform and a framework where a number of collaborative processes can be managed.

\section{OPEN SERVICE-ORIENTED PLATFORM}

Currently there is a lack of a general and adaptable framework able to grasp or integrate the various contributions to inter-enterprise collaboration, mainly those from the electronic business area that have grown up in consequence of the appearance of the XML language and web services.

There are a number of initiatives like those promoted by OASIS (Organization for the Advancement of Structured Information Standards), WS-I (Web Services Interoperability), OAGi (Open Applications Group, Inc.), STAR (Standards for Technology in Automotive Retail), Odette (International Automotive Industry Organisation, initially "Organisation for Data Exchange by Tele-Transmission in Europe"), ITA (Information Technology for the Automotive Industry), AIAG (Automotive Industry Action Group), to mention only a few organisations that are mainly concerned with collaboration in complex scenarios and are led by different industry sectors (potential end-users). Another group of organizations that are more technology-oriented include W3C, IETF, WFMC, BPMI, OMG, WS-I, which are mainly concerned with technological interoperability. Even if there is a trend towards the predominance of a limited number of leading paradigms, mainly when 
the industry leading companies are involved, most of the developed frameworks are not generic enough to support evolution and are not independent of some vertical industrial sector. As an example, the OAGi organization is defining a set of BODs using accepted languages like XML, XML Schema, and WSDL, to generate message templates to be adopted by cooperating partners. It is however complex to maintain such templates or message rules embedding semantic details, and difficult to evolve and support innovation processes when it is necessary to establish disruptive changes in terms of technology and business levels. Furthermore, the process definitions and the execution platform bindings, an important asset of enterprises, are difficult to move to another execution platform as it requires heavy development efforts (Figure 1).

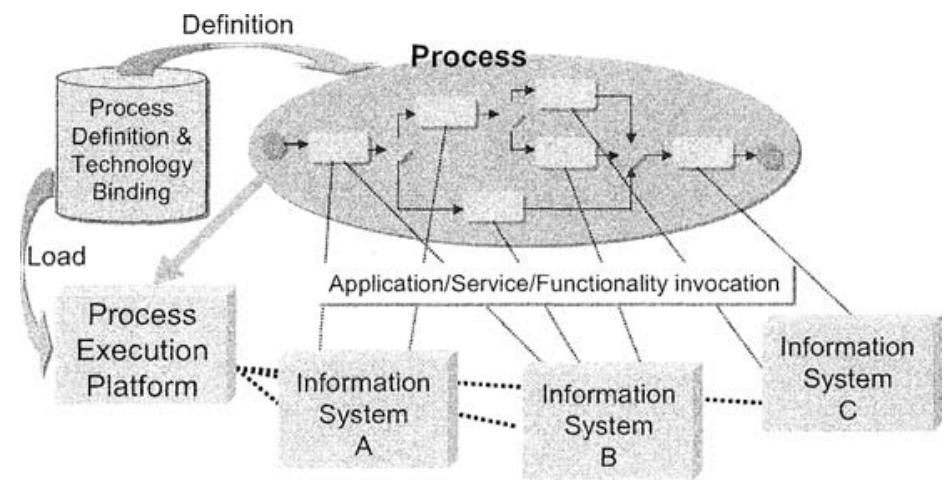

Figure 1 - Portability of process definition and technology bindings among platforms

Existing industrial solutions do not offer a clear strategy on how to move a process definition and technology binding developed for a specific execution platform to another competing platform. The Apache project Beehive (beehive.apache.org) is a contribution in this direction by providing a meta-programming model for J2EE, making easier the access to enterprise resources and other model-driven development facilities. The Controls concept offered by this project is: " $a$ lightweight, metadata-driven component framework for building that reduces the complexity of being a client of enterprise resources. Controls provide a unified client abstraction that can be implemented to access a diverse set of enterprise resources using a single configuration model." This concept offers an integration layer unifying the invocation from the activities of the process to the enterprise resources (files, services, components). However, this open initiative addresses mainly developments bound to the Java world and does not present a clear "technology agnostic" perspective, or some general enterprise resource invocation framework.

Beyond this lack of portability for process definition and technology bindings among different execution environments, a platform to support collaborative processes among enterprises is a further challenge considering the underlying diversity of cultures (different platforms, programming languages, localization languages, systems, methodologies). Consider the scenario of a company B that has adopted ebXML (Electronic Business using eXtensible Markup Language) for its 
interactions with another company $\mathrm{A}$. If a company $\mathrm{C}$ needs to cooperate with a company $\mathrm{B}$ that has adopted ebXML business framework (Figure 2), the solution is not easy and involves an adaptation (effort) investment. This investment might range from the business level to the technological level, by crossing a number of other problem domains like the organizational one.

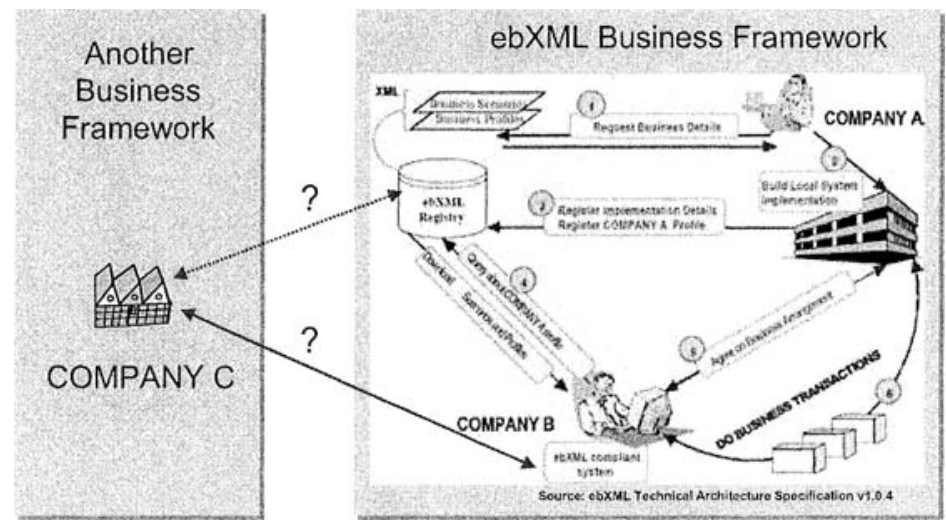

Figure 2 - Challenge for the interoperability between two business frameworks

A strategy grounded on a generalized consensus is not a realistic solution as it happened with the CORBA distributed objects framework from OMG. Even if CORBA continues to be important as one distributed object platform, it coexists with other equivalent technological approaches, like .NET distributed objects. Considering the fast evolution of ICT, the strategy must involve the coexistence of heterogeneous approaches, be them industrial, academic or open community contributions. However, it is important to have some common interoperability facilitators. There are some technologies that are the key to a crescent level of integration namely the XML language and more recently the web services technology with its origin in SOAP (Simple Object Access Protocol), initially developed to overcome firewall problems in distributed applications. The utilization of HTTP and the port 80 usually opened to access enterprise web servers, was envisaged as a prominent tunnelling mechanism to interconnect distributed applications based on remote function calls.

Therefore, in addition to the interoperability problems at technology level, a main concern is the definition of an open service bus based on agreed interfaces and contracts. This bus can be seen as an abstract service layer that is the basis for the functional binding and execution support for processes. It should be based on Web Service standards like WSDL and XML Schema, and supported on a common ontology targeted to each specific application domain. Collaboration scenarios, as described in the next chapter, require the definition of a services' ontology and (interfacing) meta-model entities in order to make possible the dynamic adaptability of services developed by different suppliers.

For most companies, the openness of systems and services regarding multivendor solutions is fundamental in order to keep a balanced positioning in the market offer. This requirement establishes a challenging research topic i.e. how to 
ensure the portability of collaborative process definitions among heterogeneous execution platforms. This portability should be grounded on the diversity of (multivendor) execution platforms existing in the various network members, and thus contributing to a paradigm shift on the practice of enterprise collaborative solutions.

\section{PROCESS BASED COLABORATIVE ENTERPRISE}

In a wide range of application scenarios, processes are the core element of a collaboration framework. Collaboration acts ranging from a simple dialog between two persons to a complex business interaction among a group of partners can be modelled as processes. A (collaborative) process can be the central modelling entity to represent collaboration acts in the context of a virtual organization or any other more traditional collaborative network.

Case 1. As an example, a new generation of electronic toll collection (ETC) systems based on the Intelligent Transport Systems Interoperability bus (ITSIBus) was implemented for the Brisa motorway management company (Osório et al., 2003). The underlying technological infrastructure is based on services with an open interface and contracts. The functional units are implemented as services and some of them are executors of process definitions promoting a shift from a hard-coded business logic to a more flexible and adaptive declarative representation of business logic. In the developed approach, a service layer (Figure 3) establishes an open service bus were a service can advertise its functionalities and events and lookup other services and event based on a discover/advertise mechanism.

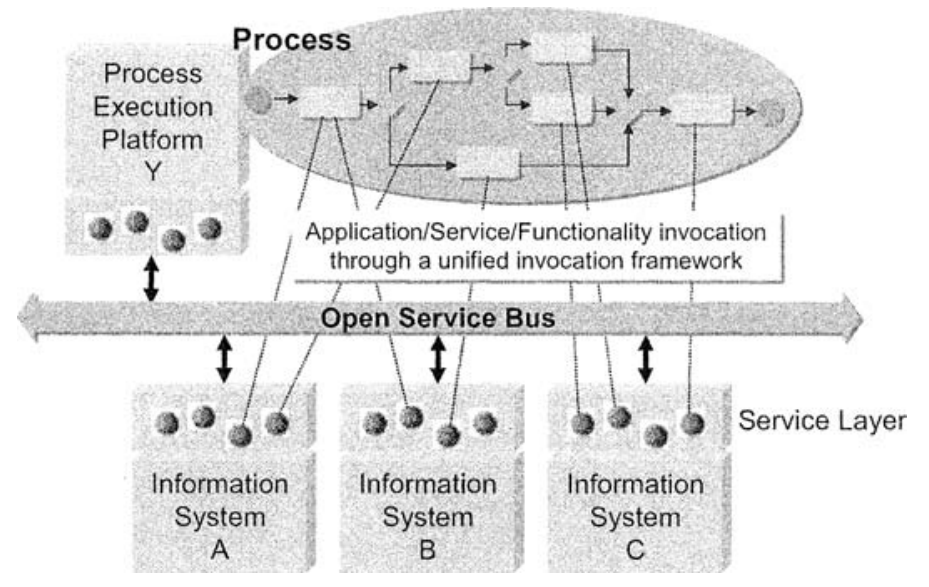

Figure 3 - Unified invocation framework for enterprise resources

In this example, a trend towards a model driven process management system is assumed. But this strategy shows the need for a reference architecture able to establish open meta-models characterizing each contributing service and providing at least three main systemic views: the design view, the operational view and the management view. At design time, there is a need for a new generation of tools able to support a model driven approach to solution construction, test and deployment. 
The existing development tools are not prepared to deal with a collaborative process and service-based solution design. There is a need for service interface and contract representation (graphical), collaborative process design and a set of tools to simulate execution and debugging of such complex solutions.

The ITSIBus architecture establishes the concept of system as an execution container running a set of services, as minimal execution entities - computational agents, sharing common environmental facilities like plug-and-play, monitoring and security (Osório et al., 2005). These services can range from simple business logic to intelligent agents, embedding logic to act autonomously in situations where central coordination is not available or decision making is necessary. When playing the role of adapter to legated systems, services can be implemented by intelligent agents as proposed by (Suh et al., 2005) with its collaboration agency architecture. In Brisa's ETC domain processes are well structured, considering that system behaviour is deterministic and defined at process definition phase.

Case 2. However in other application cases the situation can be quite different. As an example, let us consider the case of collaborative networks in e-government, the Citizen Shop case. The goal here is to establish a platform that allows offering integrated services to the citizen on a single attendance point (the "shop"), independently of the governmental organizations that are actually involved in each component of the service. In this case, processes are frequently ill-structured as many situations that could not be fully determined a-priori happen during the interaction with the citizen. In other words, the "process" is composed "on the fly" depending on the specific requests and context. In these situations it might be interesting to explore a meta-model strategy as a basis for cooperative process model definition (Rolland et al., 1998) to cope with decision-oriented products (services). By registering (tracing) collaborative acts not previously defined as a deterministic process can provide a base for future construction of new process definitions by data mining.

At the current stage, the focuses of this work is on supporting processes that can be completely defined a priori. For those classes of collaborative processes, there is a "process owner" representing the network member that defined it. The process participants are services that run autonomously or on behalf of users that belong to one enterprise member of the network (Figure 4).

This "collaborative networked organization - open service bus" (CNO-OSB) platform being developed allows for a flat group of services to be orchestrated according to a number of collaborative processes definitions (service choreographies) and executed. In each enterprise member, there exists a special service with the responsibility of orchestrating distributed processes instances execution. Many instances of this process can be running in an enterprise member each one responsible for the execution of a number of process instances. This distributed and collaborative process execution platform is designed to present the following characteristics:

- Process definition establishes an activity plan where each activity involves a set of operations or a sub-process with associated meta-data to handle invocation of operations or sub-process (local or in another network member);

- Process definition, service interfaces and contracts, and event management for asynchronous operations management following standards like BPEL, WSDL, WS-Events in a tight connection to WS-I interoperability profile standards; 
- Transaction management, security, authentication and authoring, execution monitoring (instrumentation) and other functionalities necessary for a dependable distributed process execution.

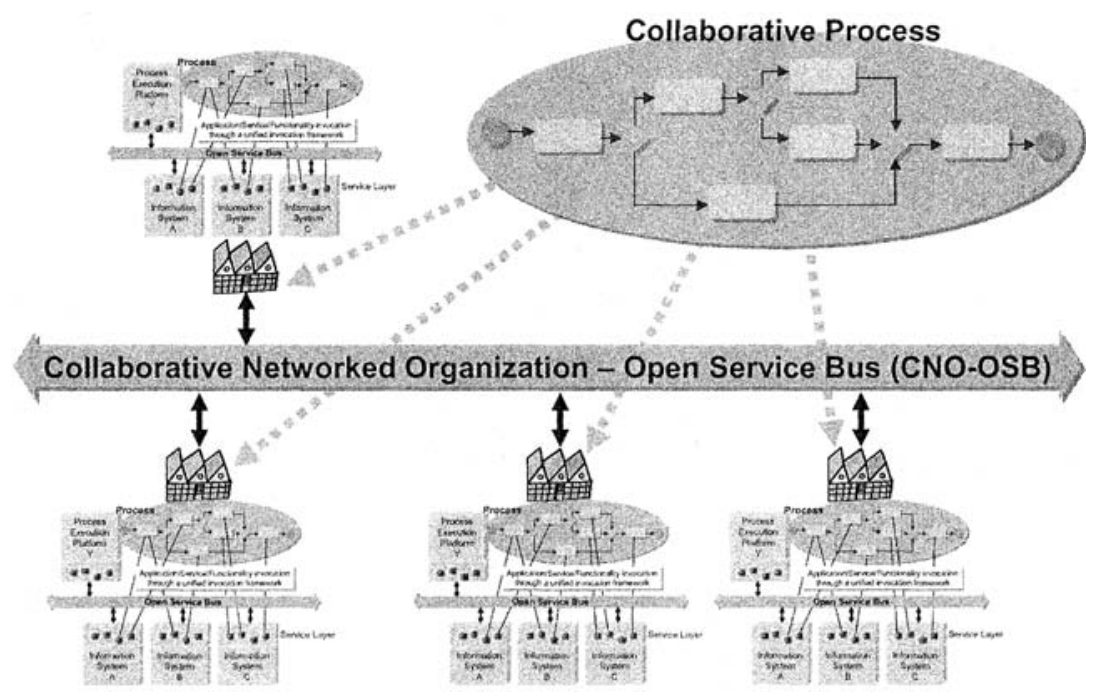

Figure 4 - The collaborative networked organization open service bus (CNO-OSB)

There is another aspect in the proposed approach and that derives from the two application examples, related with the development of a set of tools aiming to offer process experts a simple language, graphical if possible, able for them to contribute for the different life cycles of distributed processes and services based solutions. This aims to cause a shift from a technological cantered development for a process experts centred one. The idea is to work on a new model driven development approach considering developments at model level, deeply based on meta-data, independent from different technological bindings. The challenge is to promote process definition portability among competing technological platforms, creating a competitive market where today proprietary patterns prevail. To some extent, the objective is to follow a strategy established by OMG with the model driven architecture (MDA) initiative where the main objective is to generate a new set of tools developing models for technology independent platforms (PIM - platform independent models) and later bind those generated models to specific platforms (PSM - platform specific models) (Osório et al., 2005).

\section{CONCLUSIONS}

This paper discussed a distributed process execution platform for collaborative networked organizations based on the requirements from two application projects, one in the private sector and another one involving public institutions. That first case targeted the implementation of an open service-based management infrastructure for toll and traffic management. The second example aims at establishing a platform to 
offer integrated services to citizens, based on a single attendance point for a number of services executed in different public entities.

In both projects the need for establishing a collaboration culture supported by a flexible ICT infrastructure based on agreed services and contracts was identified. Although from a scientific point of view there is not much innovation in the required platform, there is a need for a pragmatic consideration of interoperability and implementation issues that take into account both existing legacy and the need to move to new business practices. In the proposed system, collaboration acts are modelled as processes, which can range from the support to a simple interaction between two persons to a complex business transaction among a group of partners. In this context there is a need for a number of modelling concepts and mechanisms that allow for the definition of collaborative processes that are able to cope with running exceptions and can easily be bound to different running platforms.

\section{ACKNOWLEDGEMENTS}

This work is partially supported by BRISA company, through the research and development projects ITSIBus and by the Citizens Shops institute (IGLC - Instituto para a Gestão das Lojas do Cidadão) through another research project aiming to develop integrated services involving several public entities.

\section{REFERENCES}

1. Camarinha-Matos, L. M.; Afsarmanesh, H.; Supporting Infrastructures for New Collaborative Forms, in Collaborative Networked Organizations, pg. 175-192, Kluwer Academic Publishers 2004.

2. Camarinha-Matos, L. M.; Afsarmanesh, H.; Ollus, Martin; Ecolead: A Holistic Approach to Creation and Management of Dynamic Virtual Organizations, IFIP International Federation for Information Processing, Pages: , 3 --16, Volume 186, Springer 2005.

3. Dillenbourg P., Baker M., Blaye A., O'Malley C. - "The Evolution of Research on Collaborative Learning"', in P. Reimann \& H. Spada (Eds), Learning in humans and machines. Towards an interdisciplinary learning science, 189-211. London: Pergamon, 1995.

4. Hollingsworth, David; Workflow Management Coalition: The Workflow Reference Model, Doc. $\mathrm{N}^{\circ}$ TC00-1003, Document Status - Issue 1.1, 1995.

5. Osório A. L., Abrantes A. J., Gonçalves J. C., Araújo A.; Miguel J. M., Jacquet, G. C.; Gomes, J. S. Flexible and Plugged Peer Systems Integration to ITS-IBUS: the case of EFC and LPR Systems, PROVE'03 - 4th IFIP Working Conference on Virtual Enterprises, published by Kluwer Academic Publishers, 2003.

6. Osório, A. Luís; Camarinha-Matos, L.M.; Gomes, J; A Collaborative Networks case study: 'The extended "ViaVerde" toll payment system, PRO-VE'05, Valencia - Spain, 26-28 September 2005.

7. Rolland, C.; Nurcan S.; Grosz, G.; A unified framework for modeling co-operative design processes and co-operative business processes, in the Proceedings of the 31st Annual Int. Conf. on System Sciences, Big Island, Hawaii, USA, 6-9 Jan.98.

8. Suh, Y. H.; Lee K. W.; Kim H.; Collaboration Agency Infrastructure for Virtual Enterprises, Proceedings of the Ninth Int. Conference on Computer Supported Cooperative Work in Design Coventry, pages 56-61, UK, May 24 -26, 2005. 